\title{
SOME RARE FORMS OF HEMATOMA.*
}

BY J. GARLAND SHERRILL, A.M., M.D., I.OUISVILLE, KY.

Our attention has been attracted recently by three cases of hematoma which continued for a much longer period than is usual in such condition, presented some difficulty in diagnosis and gave no clear explanation for their persistence except perhaps in one, where the patient was a bleeder.

In the literature on the subject we find an abundant amount of material upon hematomata in the pelvis and about the genitalia, but a great paucity of reports of cases in other portions of the body similar to the ones we report. We can account for this apparent lack of interest either because of the frequency of occurrence of hematoma, such cases have been passed over with little attention, or from the real infrequency of occurrence of cases of long standing. Or, again, the difficulty of correctly explaining their pathology may have deterred any one from taking up their investigation.

It seems to us that such cases afford some food for study in the hope of determining, if possible, the reason for their slow but steady development and their failure to follow the usual course of blood effusions. In order to determine these points we must call attention to the changes which usually follow a traumatism with the extravasation of blood in such an amount as to produce an hematoma. Hemorrhage will usually cease in a short time if the vessel be not of too great size, partly from the result of pressure against the surrounding tissues, partly from retraction and contraction of the vessel walls, and finally from the inherent poiver of coagulation of the blood. The fluid portion of this clot is normally absorbed in the course of a short time, then the corpuscular elements undergo degenerative change, a portion being absorbed and the residue remaining as pigment or hematoidin. In the presence of infection resorption of the clot does not occur, but disintegrates and forms pus. In certain portions of the body, as in the serous cavities, neither of these courses obtain, but the blood remains fluid indefinitely. The exact cause of this fluidity has not been positively established, but it is supposed that the endothelium of these sacs contain or secrete a substance capable of preventing coagulation, and that these cells perhaps have a property similar to that ascribed by some to the endothelium lining the blood vessels.

Subsequent to the absorption of the clot other changes may occur in its locality resulting in the formation of a fibroid or other form of neoplasm. A. Hoffman, in 1895, remarks that fibroma as well as other neoplasms of the abdominal wall are usually traced to some form of injury in which bloody effusion has taken place, and describes among tumors of this class a case of hematoma which had existed for seven years, as follows:

"Mrs. S., 63 years, came under treatment for gastric catarrh. On percussion of the epigastric region an unusual dull resistance was detected beginning from the lower border of the liver and extending downwards. Close inspection of the abdomen showed a flat arching to the right of the umbilicus extending upwards and gradually fading laterally. The tumor was distinctly limited to the linea alba. Bimanual examination showed its dimensions to be $7-8 \mathrm{~cm}$. by $10 \mathrm{~cm}$. As the abdomen was very flabby through previous five pregnancies it was easy to grasp the tumor within the palpating fingers.

"The history was that she felt a sudden, stabbing pain on the right, about 7 years ago, whilst threshing, as if something had burst within the abdomen, and upon feeling the part

*Read before Southern Surgical and Gynecological Association, in St. Louis, December, 1908. 
she noticed a swelling about the size of a pigeon's egg.

"The entire character of the growth points to a fibroma having its origin in the right sheath of the rectus muscle, superinduced by the original hematoma.'

W. W. Van Arsdale reports an ossifying hematoma (Annals of Surgery, 1893, Vol. XVIII) which developed within six and onehalf weeks after injury to the muscles of the arm. Dissection to the bone between the biceps and the brachialis anticus exposed a mass of apparently solid bone, continuous with the humerts, about 9 centimetres in length, and 3 centimetres broad and high. This bony mass had taken the place of the intra-muscular ligament or septum, and had invaded the origin of the brachialis anticus, so that the fibres of the muscle adhered directly to the new growth, and it was consequently difficult to say where the muscle ended and the bone began. The periosteum could not be lifted off from the tumor with the muscles attached, but appeared continuous with it; nor was it possible to say where the periosteum ended and the bone commenced. The chisel entered with some difficulty through the ossified external coat of the tumor, where was nearly $1 \frac{1}{2}$ centimetres in thickness, into a cavity; and this appeared filled with partly coagulated blood of a clark brown color; at the bottom of this cavity the bone was porous and bled on being scraped." He says, "It is true, and I am fully aware of the fact, that the handbooks of surgical pathology do not teach that a hemorrhage of the kind here reviewed can ossify," although Heinecke says: "Large encapsulated extravasasations beneath the periosteum of a bone still growing, may become enveloped with a bony capsule, as in the newly born."

Hennig (Monatsch. f. prakt., Thierheilk, Berl., 1899, 00, XI, 481), in an article upon "Hematoma in the Horse," quotes freely from a number of authors in explanation of the pathology of this condition:
"According to Billroth and V. Winiwarter, hematoma is caused by kicks or blows. The rupture which follows this crushing extends not only to the blood-vessels, but also to the lymphatics. There will be then a collection of blood and lymph in the tissues. The more vascular the parts involved and the greater the force of injury, the greater will be the extravasation, especially where a larger artery or vein is involved.

"Gussenbauer agrees with the above and thinks that these extravasations are really a mixture of blood and lymph, as there is separation of continuity in both blood and lymph vessels, necessarily followed by outpour of the contents of each.

"La Motte and Pelletan hold that these extravasations are a form of serum and compare the process to that of allowing blood to form a coagulum when placed in a vessel.

"Velpeau is of the opinion that the subcutaneous tissue is injured in such a manner that a collection of serum takes place between the aponeurosis and the general wall and compares it to a serous cyst.

"Morel-Lavallee treating on this subject, says that the tearing of small vessels and capillaries permit only the passage of the fluir components of the blood from the crushed ends. The oozing thus taking place, like in any other wound, consists mainly of a collection of the red blood serum.

"Hertwig states that the tissues involved when a trauma is inflicted offer a certain amount of resistance by tension, which causes a weakening and as a consequence blood or blood serum trickles through the vessel-walls or the lymphatics into the surrounding tissues.

"Semmer says hematoma occurs after injury of the blood vessels in parenchymatous organs or in the solid membranes. The blood is at first liquid, but later on coagulates. After breaking down and resorption of the blood which has been poured out, the hematoma disappears entirely, or it leaves a cyst filled with serous fluid, or a pigmented cicatrix. 
"Hoffman describes two cases which he considers to be lymph extravasations. In the first case he made an exploratory puncture on the second day. A bright red, watery fluid escaped and upon enlarging the opening with a lancet, from $1 \frac{\mathrm{r} / 2}{2}$ to 2 litres of the same fluid followed. This became purulent in a few days. The second case was one of tumor at the outer surface of the right thigh, with its largest dimensions over the glutei muscles. The same results followed its incision.

"Frohner denies the occurrence of lymph extravasates and says that fluctuating tumors resulting from traumatism are true hematomata. He points to the fact that the crepitation which is noticed at times upon palpation is due to the coagulated blood within the tumor."

The following hypotheses may be offered as possibilities in explanation of this condition:

1. That the blood might flow through a sac returning into the vessel, and remain fluid at least in part, just as is the case in aneurysm.

This proposition would seem to be fallacious because these hemorrhages are probably venous, and the tendency of a vein is to collapse immediately upon injury, and, moreover, no such patent. vein has been noted in any of these cases.

2. That the cellular tissues possess the power of secreting a substance which tends to prevent coagulation.

This seems clearly refuted because the infrequency of the occurrence of persistent hematoma would appear to prove the lack of such power of secretion.

3. That slight infection, present in insufficient amount to produce suppuration, results in such change that the blood cannot be taken up by the absorbent vessels.

This theory seems to us to be irreconcilable to the facts in the case, for effused blood has but little power of resistance, and any culture of bacteria should find here an excellent pabulum, and suppuration would be inevitable.
4. That the tissues about an hematoma might be so dense or so compressed that the absorbent vessels would be occluded or obliterated, thus preventing the accomplishment of their normal functions.

This proposition seems to us to have a more probable foundation in fact, although not accounting fully for the phenomena.

5. That coagulation does take place, but when disintegration occurs the blood remains fluid but not absorbable.

This contention seems also within the range of probability.

6. That the constant escape of fresh blood occurs in a quantity sufficient to more than make up for the loss in volume from absorption.

In proof of this we believe that it can be demonstrated that the escape of fluid into an hematoma does occur as is shown in my cases, in which the tumor increased in size steadily. In one the bleeding occurred from a wounded vessel in the femur; in another the source of the blood could not be discovered, but we thought it came from a small vein.

There is also the possibility of blood being manufactured inside the sac, as in the case of the development of the circulatory apparatus in the embryo, although to us it seems highly improbable that this could occur in adult life.

We conclude, therefore, from the evidence at hand that the tissues about an hematoma, from pressure lose their power of absorption; that even though coagulation and disintegration do occur resorption does not result, and that in some instances at least there is a continual addition to the amount of blood in the sac by an outflux from the damaged vessel.

In this connection we will report three cases, which, while not identical in their clinical course, present some symptoms in common:

Case No. 1. Frank R., white, 38 years; saloonkeeper and ex-baseball player. Was referred to us in 1902, at which time he presented a large growth over the right hip which 
was increasing steadily in size. The trouble began ten years previously, and appeared as a small nodule which his attendant thought to be a fatty growth, but which the patient attributed to a bruise while hunting. He claimed that this injury was received in sliding over the rail fences. He refused operation at this time.

He was seen again in January, 1904. The growth at this visit was considerably larger than when I saw him before, and was 28 inches in circumference; 10 inches in length, and 7 inches in wirlth. It was painless and quite firm, but elastic and not very hard. It creaked slightly when moved under the hand, and seemed to spring from the deep fascia covering the muscles in the gluteal region. It lay over the hip joint, but was not attached to the trochanter. Large venous trunks were seen ramifying over its surface, and all the veins of that limb were markedly varicosed. It did not move freely, although there was no

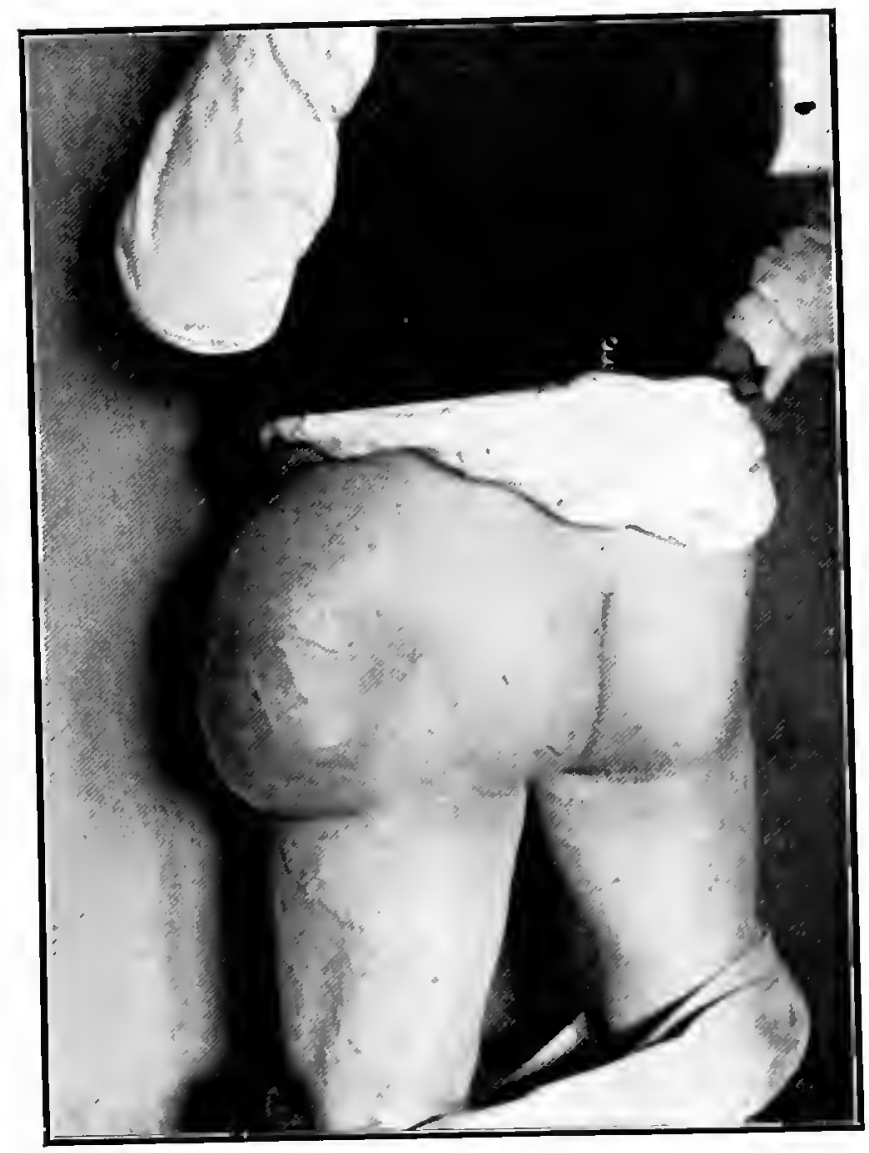

Case No.1-Hematoma-rear view.

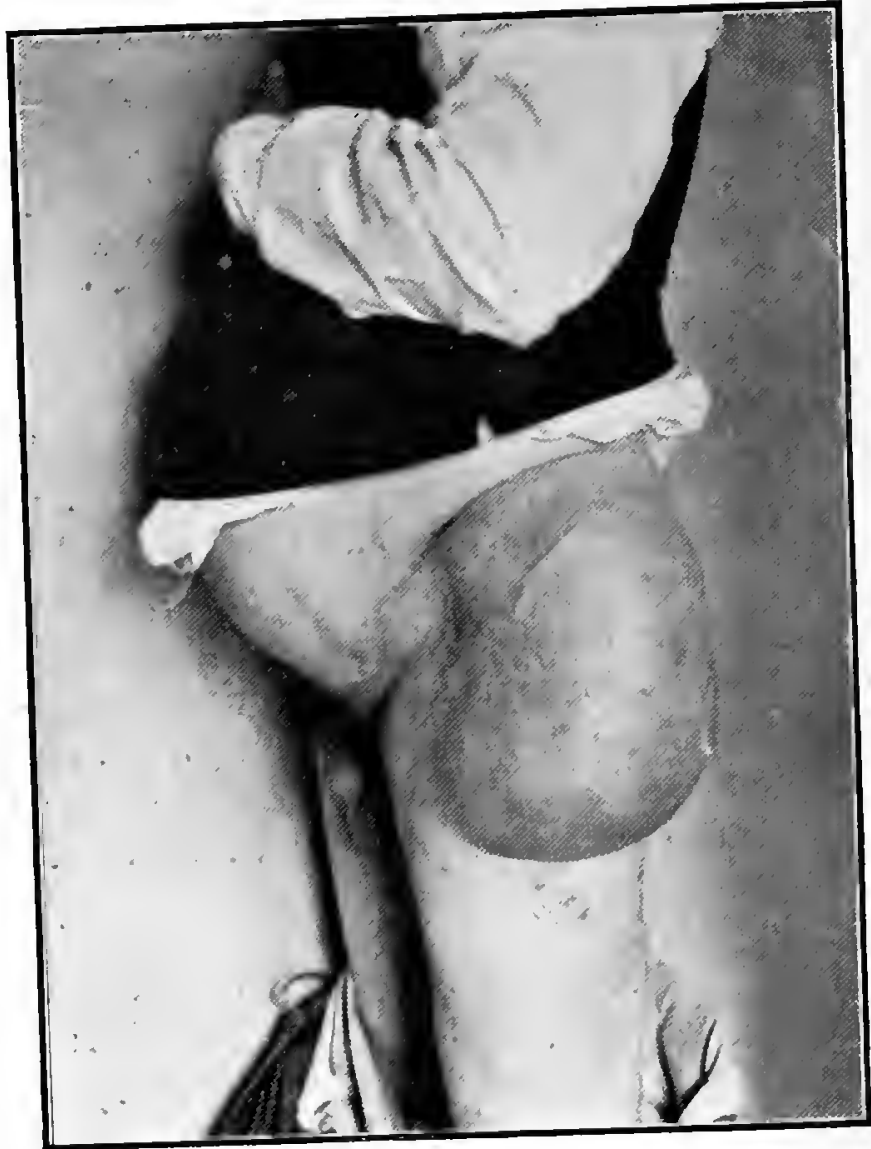

Case No.1-Hematoma-side view.

infiltration beyond its capsule and no glandular involvement. From the firmness of the tumor there was but little suspicion of its condition being a cyst. The diagnosis of a probable benign growth of fibrous tissue type was made. Sarcoma was excluded by the length of time which the condition had existed, although its appearance and vascularity made it very closely resemble one. The patient's health was not at all impaired.

In January, 1905, the growth was considerably larger, but not less movable than before. It was then 32 inches in circumference; 14 inches long, and also 14 inches across from base to hase and 8 inches across superficially.

It was removed on January 18, 1905, and was found to be composed of a rather thick fibrous tissue wall, the inner surface of which was quite smooth, the cavity containing a large quantity of fluid blood somewhat changed in color. There was no marked evidence of clotting, although there was some brownish 
deposit along its wall. The microscopic examination showed the condition to be one of simple hematoma, and Dr. John E. Hays, who made the examination, reports as follows:

Macroscopical Findings._." "This tumor contained numerous cysts or cavities filled with blood and fibrin. Across the cavities-the larger ones-extended firm bands with smooth, glistening surfaces. All the cavities examined seemed to communicate. The wall of the tumor was very irregular in thickness.

Microscopic Findings._- "The wall of the tumor consisted of rather dense white fibrous connective tissue, well formed and showing no signs of cellular activity. The bundles of fibres were separated by an infiltration of blood cells, sometimes only a single row of cells separating the fibres; then again there would be a larger number giving the appearance of small cysts. The inner surface of the walls was covered by layers of fibrin, but nowhere could an epithelial or endothelial lining be made out.

The bands or trabeculæ had the same structure as the wall.

Case No. 2. Mrs. H. W., white, 50 years; April 2, 1907. History of having given birth to nine children; always remained well until January, 1907, when she noticed a mass in the abdomen. Some time previously in carrying a tub of ice. she had fallen with the tub on top of her. Had suffered some from the bruising, but was not seriously inconvenienced by it. The mass in the abdomen had increased in size rapidly since she first noticed it, and at this time was larger than a man's head. She was a very fleshy woman, and her weight had remained stationary. She had suffered some pain in the region of this growth but had no serious discomfort, although she was somewhat worried as to the outcome of the condition.

Examination revealed the abdomen to be quite prominent, near the umbilicus and on the left side. A palpable mass presented in or adherent to the abdominal wall, extending well to the left of the median line, and also slightly to its right. It was separable from deep structures, but not from the skin, and was dull on percussion surrounded by tympany at the edges. It was not fluctuant and there was no palpable mass in the pelvis.

A positive diagnosis was not made. Hematoma, sarcoma, and cyst of the urachus were considered to be among the probabilities. Urinalysis: Specific gravity, 1008; no albumen ; no casts; no sugar. Blood examination showed number of leucocytes to be 7500 ; red corpuscles, 5,000,000; hæmaglobin, 70 per cent.

She was a dangerous anesthetic risk owing to an irregular and intermittent heart, but the operation was safely performed under gasether anesthesia, although she gave the anesthetist considerable annoyance and alarm. The condition proved to be an hematoma of the rectus sheath containing partly fluid and partly clotted blood. The cavity was packed with gauze for a few days, and the convalescence was uninterrupted.

Case No. 3. Robert K., white, 15 years. This boy was seen on the 7 th of August, 1908, with Dr. T. E. Chapman. He presented a history of an injury to the left thigh some two or three weeks previously. I found a slight swelling on the outer and anterior aspect of the thigh, which we considered to be an hematoma resulting from the injury to the limb. Advised the usual measures for its treatment.

He came to us again on October 5, 1908, having been seen by a number of other physicians, some of whom had made the diagnosis of sarcoma. They had arrived at this conclusion after aspirating the tumor and obtaining only a little blood, and an X-ray examination having failed to show any evidence of disease of the bone. A very small incision had also been made into the tumor down to the bone, which was reported to me as having disclosed only that the bone was slightly damaged.

The tumor upon his thigh had increased 
somewhat in size since I last saw him. I found on examination the mass to be at this time about the size of an orange, apparently not intimately connected with the bone, but to be attached to the deep muscles and apparently moving with the soft parts over the bone. No fluctuation could be clearly marle out, although it seemed to be cystic. The incision which had been made was not yet quite healed. The suspicion of sarcoma was so strong that I prepared to have a frozen section examined if it proved to be a tumor, although I still believed that it was an hematoma, basing my opinion largely upon my former examination and the history obtained at that time.

Operation October 7, 1908, revealed the condition to be an hematoma. After the clots were removed we found a bare spot on the bone about the size of a nickel, from which the blood was oozing. Careful examination revealed several spicula of bone; as the result, evidently, of contusion at the time of the injury, which caused an incomplete fracture. The bone bled more freely than is usual; otherwise the condition was satisfactory. The persistent hemorrhage was controlled by gauze packing and the wound closed.

On October 10, 1908, the gauze pack was removed and apparently no hemorrhage was excited at the time, but during the night following a persistent oozing occurred and the sac was again filled with bloot. This was again removed under chloroform on the day following, and the wound again packed very carefully. On close questioning this boy was found to be a bleeder, having had a number of attacks of severe hemorrhage from very slight traumatisms, and this undoubtedly in part accounted for the persistence of the blood tumor. Subsequently he made rapid progress to recovery.

We have also seen several cases of blood cysts in the neighborhood especially of the knee joint, which we considered as hemorrhages into bursal sacs near the joint, but which we did not consider as belonging in the class of cases here recorded, although in the literature upon this subject we have found mention of such cases.

The following cases have been collected from the literature, which, as we have stated before, is not very voluminous on this subject :

Nágel, L., Zeitschr. f. Wundarzt, u. Goburtsh, 1866-67, XIX, 180.

1. Boy, 6 years, had a small blood cyst, size of a small hen's egg in the right inguinal region, for which there was no assignable cause, which became inflamed and painful. As it showed some fluctuation it was lanced. No pus followed, only blood, resulting in collapse of the cyst. Length of existence not stated.

2. Man, 50 years, had a tumor on the anterior aspect of the knce, fluctuating, but not inflamed or painful, and was brought on by kneeling, his occupation being that of a mason. On opening the same the quantity of blood was so great he became faint and compresses had to be applied to check the remaining collection: No further history given except that he was dismissed from the hospital on the ninth day.

Auvard, Bull. Soc. Clin., Par. 1861, V. 98. Case of man, 52 years, with hematoma of right arm, size of two fists, situated at the antero-exterior surface. He first noticed a small swelling, 5-6 cm., about a year ago, appearing without appreciable cause. No pain or inflammation, but a weakening of the entire arm for which he came under treatment. General health good. The tumor shows fluctuation in all parts. He gave a history of spitting blood when 9 years old; typhoid fever without hemorrhages at 25 , and passage of blood per anum at 30 , but denies hemorrhoids. The author calls this, a case of "spontaneous" hematoma, as there is no traumatism connected with the history. He removed the cyst. Recovery. 
Combeleran. Toulouse Medical, 1905, VII, 78 .

Reports two cases of hematoma with fever. The first, man, about 40 years, fell down and struck the outer surface of the right thigl. Was able to walk for four days after the accident, when he was seized with violent pain in the part accompanied by swelling of both thigh and knee. The pain increasing he entered hospital two days later. No signs of fracture could be detected, but some effusion into the knee-joint. For three days the temperature oscillated between 37 degrees and 38.8 degrees. On the fourth day it rose to 39.2 degrees. At this time an incision was made over the external aspect of the thigh and a large quantity of blood was evacuated. Temperature, 40 degrees; suppuration of the hematoma; several days of cirainage and repeated incision eventually led to a cure.

The second case was in a tabetic subject, who in making a false step fractured the left femur at the middle part. The day following liis temperature was 38 degrees, and a voluminous hematoma was detected in the thigh. The temperature did not fall for 6 days. No further details are given.

This writer says these two cases are interesting as experimental and clinical facts show that elevation of temperature is not always due to infection, but to the resorption of certain organic substances. Experiments on animals demonstrate that blood, injected into an animal of the same or different species, causes a rise of temperature, whether done subcutaneously, intra-venously or into a serous cavity. Clinically, the above cases showed fever following immediately the effusion of blood. Opinions are divided as to the causes of this rise of temperature. Some hold that the white corpuscles are increased or altered. The same difference of opinion exists as to the causes of resorption. Is it the leucin, the fibrin ferment or the nuclein?
Lemariguier, A., These, Paris, 1886.

Case 1. Clerk, 54 years, fell down the stairs, striking his left thigh, but continued his occupation as he was able to move about without great inconvenience. Ten days later he noticed a swelling the size of a fist, painless, except upon strong flexion of the knee, which rapidly increased and infiltrated the sub-sponeurotic parts of the lower portion of the thigh. Considerable pain and inflammation now set in, with spontaneous rupture of the sac several days later. Recovery.

Case 2. Leather dresser, 43 years, hematoma of left clavicular region. Seven months before, in carrying a lieavy load on his shoulder, he suddenly felt a crackling at this site, and next day noticed a swelling about the size of a nut, without ecchymosis or pain. Within the last two months it had attained the size of a hen's egg.

Diagnosis: Lipoma, cold abscess or hematoma. On incision about 100 grammes of a syrupy, chocolate colored fluid was evacuated and the walls of the cyst extirpated. Further examination revealed its true nature. Suppuration followed the operation, but recovery gradually took place.

Case 3. Man, 33 years, reported by M. Follet to the Surgical Society, February 25, 1885, that 20 years before in trying to raise himself from the floor experienced a sharp pain in left buttock. Several weeks later he noticed a swelling, size of hen's egg, which gradually increased in size, until it reached that of a man's head. A small ulcer had formed and this evacuated a sanguinolent fluid, estimated at between 7 and 10 litres. Diagnosis of encysted hematoma from rupture of the gluteus maximus. Operation was done with great difficulty and recovery tedious.

He says in conclusion:

1. Traumatic bloody effusions terminate ordinarily by complete resorption.

2. Resorption is more or less slow and is 
sometimes favored by the infiltration of the blood in the neighboring connective tissue, or the accidental rupture of the cyst.

3. Leucocytes play a considerable role in the absorption of the blood.

4. Hematoma sometimes terminates by abscess, sometimes by plegmon, diffused or circumscribed.

5. The causes of non-resorption of the blood are numerous, especially the seat of the hematoma, such as the buttock, the superior and external side of the thigh, et $:$, and in the parts having abundant adlipose tissue. The amount of blood encysted is no obstacle to resorption.

Reverdin. Bull. Soc. Anat., Paris, 1868, XLIII, 612.

Hematoma of right rectus muscle of the abcomen in a woman 39 years. Was admitted to hospital September 15, 1868, with pulmonary phthisis. On September 29th she felt a sudden pain in the epigastric region, which she compared to a blow, and which was greatly increased on pressure. The skin presented notling particular. In the course of a few days it cisappeared. On November 26, during a fit of coughing she was again seized, this time with greater intensity than before. Tr. iodine was applied with but little relief; in fact, she did not respond to any of the remedial measures instituted, and death came to her December 5 . Autopsy showed the muscles of the right side of the abdomen infiltrated with blood, with inflammation of the muscle tissue as demonstrated by microscopic examination. He called it a "muscular hematoma."

McLeod. The Indian M. Gaz., Calcutta, 1880, XV. 305.

Hematoma, or Venous Aneurysm, removed from the left axilla of a male, 45 years. About two months before admission noticed a small swelling, size of a betel nut in his left axilla; it was soft, painless, and freely movable. It began to increase and in 15 days was as large as a hen's egg. In $1 \frac{1}{2}$ months it filled the axillary space, size of a cocoanut, got tense, painful and exerted pressure upon the brachial plexus. It bulges downward and the left infraclavicular and mammary region of the chest. Posteriorly it extends as far as the axillary border of the left scapula. It is bounded above by the clavicle and below by the seventh rib. Its longitudinal diameters, 8 inches, and the transverse is about a foot. The whole tumor is uniformly rounded, with a broad base; it is fixed to the chest wall. The surface of the tumor is smooth, the covering healthy and freely movable; its consistency soft and fluctuating. There is no throbbing pain, no surface edema, no increase of temperature, and no pulsation; no bruit on auscultation. There is slight deficiency of sensation in the upper and outer half of the left arm; no edema of same and no clange in the axillary or brachial pulse. The tumor was explored by a fine trocar and canula through which a free flow of dark venous blood took place and the swelling diagnosed to be a tumor containing blood.

Operation difficult and patient suffered much from shock. Recovery.

"These blood cysts are exceedingly rare and little is mentioned in the literature. It was quite certain it had not formed in a pre-existing tumor either of a sarcomatous or angiomatous nature and it was very unlikely that the cyst had originally been of a serous nature, and had contracted a communication with the axillary vein. The history did not throw much light on its origin, which was and remains a mystery."

Maunsell, R. C. B., Tr. Roy. Acad. M., Ireland, 1899, XVII, 514. on Vascular Tumors of the Abdominal Wall:

Case 1. Miss F. K., 22 years, had noticed a lump in her left side just below the costal margin, but latterly it increased in size and caused considerable pain. Examination showed an elongated tumor as large as an adult hand. 
Pressure caused great pain. The consistence of the mass varied, some parts feeling tense and some soft and lobulater; the edge was not well clefined; the skin was movable except in the center where it appeared to be adherent. Diagnosis of lipoma.

The parts were removed and consisted of adipose tissue connecting together several cysts of various sizes lined by endothelium and containing smooth muscle in their walls, considered as of lymphatic origin. She developed erysipelas by scratching the scar, but recovered in a few weeks.

This last case can scarcely be classed under the head of persistent hematomata such as we have here reported; nor can the second case of Nagel, which was apparently an effusion into the prepatellar bursa.

The cases of Combeleran, too, while interesting, should be excluded from the list. There remain then 13 cases in literature, including three of my own, having some similarity, yet cliffering in minor details. The case of $M$. Follet mentioned by Lemariguier had a duration of twenty years, while one of my cases lasted over ten years.

Traunatism was noted in all but four cases in which the bleeding was spontaneous.

We must conclude that these cases are quite rare, since the reports are so few; also that we have no exact knowledge concerning the causes leading to their persistence. We offer the conclusions mentioned before in explanation of their formation.

\section{BIDLIOGRAPHYr.}

Henning, Monatsch. f. prakt. Thierheill, Bcrl., I899-00, XI., 48I.

Billroth \& V. Winiwarter, Die allgem. chin. Path. u. Thcrap. I889.

Gussenbauer, Die traumat. Vcrlctz, I890.

La Motte \& Pelletan, Traite de Chir., T. i. p. I722.

Velpean, Arn. dc Chir., I833, VII.

Morel-Lavalee, Arch, gcner. de med., I853, i.

Hertwig, Prakt. Handb. Chir. f. Thicrarzt, I874.

Semmer, Koch's Encyk. d. Thierlhcilk, I893.

Hoffman, Monatsh. f. prakt. Thierhcilk, I 890 i.

Hoffman, A., Inaug. Dissert., Wurzburg, 1895.

Schlacht, C., Inaug. Dissert., Berlin, I884.

Nagel, L., Zeitschr. f. Wundatzt. u. Geburtsh, I86667, XI. X., I 89.

Auvard, Bull. Soc. Clin., Par. I88I, V., 98.

Combeleran, Toulouse Medical, I905, VII., 70.

Lcmariguier, A., These, Paris, I886.

Patel, M.M., La Province Med., Lyon, I900, XIV., 336.

Reverdin, Bull. Soc. Anat., Paris, I868, XLIII., 6 I2.

McLeod, The Indian M. Gaz., Calcutta, I880, XV., 305 .

Maunsell, R. C. B., Tr. Roy. Acad. M., Ircland, I899, XVII., 514 .

W. W. Van Arsdale, Annals of Surg., I893, Vol. XVIII. 